\title{
Jean Laspougeas \\ La Nation ou la Liberté? L'université de Caen contre la Révolution, 1791
}

L'assoupissement, voire la décadence des universités ${ }^{1}$, fait partie des idées reçues sur la France à la fin du XVIIIe siècle. Ces institutions se signalent par une ssclérose de plus en plus manifeste. ${ }^{2}$. Des tableaux de la France à la veille de la Révolution de 1789 ignorent complètement l'existence de vingt-trois centres universitaires. Ces centres, il est vrai, sont à la fois d'Eglise et d'Etat. Or, ce qui différencie le plus l'ancienne France de la France nouvelle issue de la Révolution est précisément son régime ecclésiastique. Comme l'Eglise catholique en France pendant la deuxième moitié du XVIIle siècle passe encore parfois comme en décadence, le désintérêt - sinon l'ignorance - peuvent trouver là une justification. Au demeurant les universités n'ont-elles pas disparu, sans trop de bruit au cours de la Révolution, à la charnière de l'an I et de l'an II de la République, avec les derniers vestiges de l'Ancien Régime? En tout cas une histoire des universités en France peut ignorer la crise révolutionnaire ${ }^{3}$. A défaut d'ouvrir un dossier général, la Déclaration publiée par l'Université de Caen, le 25 mai 1791, introduit au débat des idées politiques et religieuses, non seulement à l'époque de la Révolution mais au-delà. Comment se présente cette université en 1789-1791? Quels sont la nature et le contenu de la déclaration du 25 mai? Quelles conséquences a-t-elle engendrées sur le champ et ultérieurement? Voilà les questions essentielles qu'il faut successivement examiner.

1 Tableau très sombre dans Félix Ponteil, Histoire de l'enseignement en France (Paris 1966) et, plus récemment dans François Lebrun, Marc Venard et Jean Quéniart, Histoire générale de l'enseignement et de l'éducation en France, tome 2 : De Gutenberg aux Lumières (1480-1789) (Paris 1981).

2 Lebrun, comme n. 1, 561.

${ }^{3}$ Dans un ouvrage qui - il est vrai - ne se veut ni synthèse, ni manuel mais s'intitule cependant Histoire des universités en France (Toulouse 1986), Jacques Verger s'interroge sur la Révolution comme indice de structure profonde ou de mutations importantes (Histoire des universités, 8). La crise révolutionnaire y est purement et simplement passée sous silence, ne faisant pas partie edes périodes décisives dans l'histoire de l'institution elle-même* (Verger, Histoire, 8). 


\section{L'Université de Caen, de la réforme à la crise, 1789-1791}

En 1789 il est bien difficile de tenir l'Université normande pour moribonde, ou simplement sclérosée ${ }^{4}$. Bien complète avec ses quatre Facultés - théologie, droits, médecine, arts - elle se trouve dans une situation matérielle enviable ${ }^{5}$. Ses effectifs d'étudiants ne sont pas négligeables: trois cent quatre vingt huit inscrits en novembre $1788^{6}$. Son état général est assez éloigné de l'image qu'en donnent des cahiers de doléances confectionnés en Normandie ${ }^{7}$. Sans doute Caen n'est pas ville épiscopale, mais l'évêque de Bayeux, chancelier de l'Université, n'est pas loin et dispose d'une résidence à Caen même. Un séminaire caennais, tenu par les Eudistes, fait partie de la zone d'influence de l'Université. Sans doute Caen n'est pas ville de Parlement et l'aire d'attraction de l'Université peut sembler limitée: en 1780-89 69\% des étudiants en droit et les trois quarts des étudiants en médecine viennent de Normandie ${ }^{8}$. Mais si la peregrinatio academica n'est plus d'usage ordinaire en France à cette époque, le rétrécissement géographique de l'Université ne saurait être exagéré. De même, de la rareté des œuvres imprimées, il ne faudrait pas conclure à un rétrécissement scientifique, voire à une immobilité. Au contraire la royauté élève les exigences et l'Université de Caen se trouve, en 1789, en pleine réforme. Il est plus juste de la considérer, non comme un centre de recherche à proprement parler, mais comme un centre supérieur de diffusion des idées ${ }^{9}$, un foyer de culture religieuse et intellectuelle ${ }^{10}$, marqué par la modération, tant en droit qu'en théologie, l'institution étant membre de la structure monarchique de l'Eglise comme de la structure monarchique de l'Etat.

4 L'histoire de l'Université de Caen a fait l'objet d'une esquisse de Henri Prentout, 1432-1932. L'Université de Caen, son passé, son présent, sous la direction d'Alexandre Bigot (Caen 1932). Cette esquisse n'a pas été remplacée. Solide monographie sur la Faculté des droits par Emile Bridrey, Les dernières années de l'ancienne Faculté des droits de Caen (Paris 1931). Pour replacer l'université normande dans l'ensemble français, Roger Chartier, Dominique Julia et Marie-Madeleine Compère, L'Education en France du XVIe au XVIIIe siècle (Paris 1976).

${ }^{5}$ Avec 42000 livres de revenus, c'est une des universités les mieux dotées du royaume. En théologie et en médecine, le traitement des professeurs atteint 1200 livres par an; aux arts, il varie entre 1200 et 1500 livres, attestant les ressources du centre universitaire normand, parmi les plus élevées des universités françaises. Roger Chartier, comme n. 4, 260.

6 En théologie, 86 inscrits; avec 239 inscrits, la Faculté des droits de Caen n'est dépassée que par Paris (728) et Toulouse (420); avec 33 inscrits, la Faculté de médecine n'est dépassée que par Montpellier (219), Paris (100) et Toulouse (57); elle vient au troisième rang pour les gradés, en fournissant $8,1 \%$ des médecins de France, derrière Montpellier $(45 \%)$ et Toulouse $(9,4 \%)$. Roger Chartier, comme n. 4, 273-276.

7 Bien des cahiers dénoncent la rapidité des études de droit, le laxisme, voire la vénalité des examens. Louis Liard, L'Eriseignement supérieur en France 1789-1889, t. 1 (Paris 1888) 106-115 et Henri Prentout, comme n. 4, 181-182. Seuls sont suspectés pour leurs faibles études les notaires, les avocats, les officiers.

${ }^{8}$ La proportion dépassait $90 \%$ au XVIe siècle Jacques Verger, comme n. 3, 164 .

9 Nous suivons Laurence W. B. Brockliss dans Jacques Verger, comme n. 3, 199-253, qui corrige l'image noire des universités françaises au XVIIle siècle.

10 L'universitaire est uun chrétien fervent, Laurence Brockliss dans Jacques Verger, comme n. 3, 251. 
Rapidement la Révolution compromet la réforme en cours provoquant deux crises: une crise des effectifs et une crise de conscience. La baisse importante des inscrits en novembre $1789^{11}$ n'est pas une crise interne, mais externe. Officine de clercs et d'officiers, l'université perd de son utilité avec les nouveaux systèmes ecclésiastique et judiciaire, eux-mêmes éloignés des doléances de 1789. Loin en effet de lutter contre le laxisme des examens, l'Assemblée constituante supprime toute espèce de diplôme pour l'exercice des professions judiciaires: plus de grades pour les fonctions judiciaires, mais un certificat de civisme, cinq ans de métier et un acte de foi dans l'élection de la Nation souveraine. Faut-il s'étonner si le bibliothécaire de l'Université met en vente sa bibliothèque personnelle et se dispose à partir pour l'Angleterre? En ce printemps 1791, l'Université, il est vrai, est frappée par une nouvelle crise: la crise du serment ${ }^{12}$.

Par décret du 27 novembre 1790 l'Assemblée nationale avait astreint au serment les *ecclésiastiques fonctionnaires publics» ${ }^{13}$. Dans le département du Calvados, le directoire du district de Caen avait avisé les professeurs et régents des ordres sacrés le 12 janvier 1791: près de vingt-cinq personnes dont quatre à la Faculté de théologie, les autres étant à la Faculté des arts, principaux ou régents des collèges pour la plupart. Avant même l'ouverture des registres de prestation des serments par la municipalité de Caen, une lettre collective annonçait un refus: de facto, six ecclésiastiques prêtèrent serment dont trois de la Faculté des arts.

La crise rebondit avec le serment universitaire. Le 22 mars 1791 l'Assemblée nationale, en effet, prend un nouveau décret astreignant au serment les professeurs et agrégés des Facultés ${ }^{14}$. Le 27 avril au département du Calvados, le 7 mai au district de Caen, ce décret est enregistré comme "loi relative ... de l'Université de Paris». Entre temps, l'Assemblée constituante avait adopté un nouveau décret étendant l'obligation du serment aux universités de province. Ce décret des 15-17 avril est enregistré au département le 9 mai et au district le 16 mai. Dans le même temps le pape venait de condamner, par deux brefs (10 mars et 13 avril), le système suivi en France ${ }^{15}$. A Caen,

1173 inscrits en théologie au lieu de 86 en novembre 1788; 157 aux droits au lieu de 239; 18 en médecine au lieu de 33 . En novembre 1790 il n'y a plus que 28 inscrits aux droits.

${ }^{12}$ Mise en vente des livres composant la bibliothèque de Moisant, bibliothécaire de l'université: environ 3000 volumes, dont 200 in folio et 500 in quarto: Affiches, Annonces et Avis divers ou Journal de la Basse-Normandie et du Calvados, 6ème année, $\mathrm{n}^{0}$ 5, 27 mars 1791, 1.

13 Sur les serments et les crises qu'ils provoquent pendant la Révolution nous renvoyons une fois pour toutes à Bernard Plongeron, Conscience religieuse en Révolution (Paris 1969).

14 Article Ier: .Toutes personnes chargées d'une fonction publique dans le département de l'instruction, qui n'ont pas prêté le serment prescrit par les décrets des 27 décembre et 22 mars derniers, sont déchues de leurs fonctions; et il doit être provisoirement pourvu, s'il est nécessaire, à leur remplacement par le directoire du département, Jean-Baptiste Duvergier, Collection complète des lois, II (Paris 1824) 370.

${ }^{15}$ Le bref du 10 mars Quod aliquantum est adressé aux prélats membres de l'Assemblée nationale, en réponse à l'Exposition des principes sur la Constitution civile du clergé que trente évêques députés avaient publiée le 30 octobre 1790. Quant au bref du 13 avril, adressé à la France comme au Sacré-Collège, il condamne le système français et frappe de suspense tout ecclésiastique assermenté qui ne se rétracterait pas dans les quarante jours. A Paris, le nonce en fait mystère mais l'abbé Royou en donne une traduction française. Le bref est publié le 4 mai au diocèse de Lisieux, le 1er juin au diocèse d'Evreux, le 6 juin aux diocèses de Rouen et de Séez, le 1er décem- 
la lutte faisait rage depuis le début de l'année ${ }^{16}$. L'évêque de Bayeux, Mgr de Cheylus, naguère prélat mondain et absent, après avoir tenté un ralliement à la Révolution en devenant maire de Bayeux (printemps 1790), redevenait évêque dans toute la force du terme, mobilisant pour les droits de l'Eglise, non seulement ses forces épiscopales, mais aussi les forces capitulaires, paroissiales et monastiques. Son action, de conciliante, avait dû devenir intransigeante, obtenant des résultats à Bayeux d'abord, où la majorité des curés, naguère proches de la Révolution (8 à 9 sur 14), se sont rangés derrière lui: 12 sur 14 au début de 1791 . Grosse majorité de curés insermentés à Caen, dont le maire, Leforestier de Vendœuvre, est un des rares notables laïcs, élus dans les nouvelles institutions locales, à oser s'élever publiquement contre le serment constitutionnel. Un curé assermenté de Caen, élu évêque constitutionnel du Calvados, renonce. Pour la nouvelle élection le nombre des abstentions grandit et l'évêque de Bayeux n'a pas de peine à démasquer la personnalité du nouvel élu, Claude Fauchet. Pour échapper à une arrestation, depuis longtemps souhaitée par les milieux patriotes - autorités et clubs - Mgr de Cheylus doit s'enfuir vers les îles anglo-normandes par Cherbourg (début mai).

Dans la crise dédoublée du serment ecclésiastique et du serment universitaire ${ }^{17}$, le Tribunal du recteur de l'Université se donne une commission permanente (12 mai). Pour la Saint-Yves, patron des droits (19 mai), l'Assemblée générale de l'Université décide de se faire communiquer par les autorités locales les textes authentiques de la loi. En fin de compte, treize universitaires prêtent serment: trois aux droits, quatre en médecine et six aux arts. Entre temps, lors d'une nouvelle assemblée générale, le 25 mai, l'Université, loin d'obtempérer aux décrets de l'Assemblée nationale, avait adopté une déclaration, manifestation collective de résistance, élément nouveau d'une exceptionnelle gravité et révélateur, non seulement de la crise de conscience provoquée par l'Assemblée nationale, mais du débat intellectuel soulevé.

\section{La Déclaration du 25 mai 1791}

Longuement motivée, la délibération universitaire est immédiatement rendue publique au moyen de l'impression, sous le titre: «Déclaration de l'Université de Caen au sujet des lois des 22 mars et 17 avril dernier, concernant le serment prescrit aux fonctionnaires publics* ${ }^{18}$. Si l'occasion est bien le serment imposé aux universitaires selon

\section{Fortsetzung Fußnote von Seite 65}

bre au diocèse de Bayeux: Emile Sevestre, Les problèmes religieux de la Révolution et de l'Empire en Normandie, 2: La Constitution civile du clergé, 1791-1795 (Paris 1924) 200.

${ }_{16}$ Sur la crise religieuse en Normandie, outre Emile Sevestre, comme n. 15, voir Robert Patry, Une ville de province: Caen pendant la Révolution de 1789 (Condé sur-Noireau 1983), malheureusement dépourvu de toute référence archivistique et bibliographique.

${ }_{17}$ Bridrey, comme n. 4, 29-30 ... insiste à juste titre sur la distinciton à faire entre la crise du serment d'une part et la déclaration de l'Université d'autre part.

${ }_{18}$ Archives départementales du Calvados, série D, 83: Registre des actes et conclusions du Général de l'Université de Caen, fol.40 à 44 . Texte imprimé repris par Armand Bénet, Inventaire 
une formulation qui englobe l'œuvre ecclésiastique de l'Assemblée constituante ${ }^{19}$, la déclaration révèle que la Constitution civile du clergé n'est pas la seule pierre d'achoppement. Signée par quarante-sept universitaires (dont neuf en théologie, six aux droits, douze en médecine et vingt aux arts), elle passe pour avoir été préparée par un ecclésiastique, l'abbé de La Rue, professeur d'histoire et de géographie au collège du Mont, doyen de la Faculté des arts, et un laïc, Hippolyte Marc, agrégé des droits ${ }^{20}$. Tout en assurant les pouvoirs publics de son attachement à ses fonctions savantes et éducatives, l'Université refuse de prêter le serment universitaire prescrit par la loi du 17 avril 1791. Pour la portion stemporelle de l'œuvre de l'Assemblée constituante, les universitaires caennais envisagent tout au plus un serment restrictif. Quant à la partie ecclésiastique de l'œuvre de l'Assemblée, l'Université oppose un refus catégorique, après ceux du pape, des évêques et notamment de l'évêque de Bayeux, son chancelier, réfugié à Jersey.

\section{A. Une critique dévastatrice en forme de serment restrictif:}

Après avoir rappelé son attachement à son rôle intellectuel, l'Université accepte «également. de prêter serment à la Nation, à la Loi et au Roi, et, par suite, à la constitution nouvelle de la France. Mais en multipliant, avec alacrité, les mises au point et les définitions, la déclaration en arrive à dénoncer, et le serment prescrit aux universitaires, et la dégradation de la situation et le rôle prépondérant de l'Assemblée nationale elle-même, dont elle met en doute la légitimité.

Le serment? C'est un abus de pouvoir de la part de l'Assemblée. Le seul serment exigible des universitaires eût été le maintien de la vie intellectuelle et scolaire.

La dégradation de la situation? Elle conduit à une sanarchie sauvage et antisociale*. De fait villes et campagnes se déchirent en Normandie comme ailleurs, non seulement depuis la crise ecclésiastique qui vient d'éclater, mais aussi depuis les découpages administratifs opérés en 1790 , source - souvent négligée par l'historiographie de la Révolution - de rancœurs et de troubles. Aussi bien, depuis 1789, la sécurité des personnes reste en cause: pour échapper au parti patriote, qui voulait le faire arrêter, l'évêque de Bayeux a dû s'enfuir au début du mois de mai.

Au-delà du serment et des troubles, l'Université met en cause le pouvoir de l'Assemblée nationale, dans son œuvre comme dans sa nature.

Dans son cuvre. - La Révolution opérée par la Constituante ne peut se justifier par l'expérience. Surtout elle ne peut se justifier par l'assentiment des citoyens puisqu'elle comporte des aspects fort éloignés des doléances électorales de 1789: l'Assemblée ne

\section{Fortsetzung Fußnote von Seite 66}

sommaire des archives départementales antérieures à 1790 : Calvados, série $\mathrm{D}$, tome 2: Université de Caen (Caen 1894) 252-256.

19 . Nous jurons d'être fidèles à la Nation, à la Loi et au Roi, de maintenir de tout notre pouvoir la Constitution nouvelle du royaume, et de remplir avec fidélité et exactitude, et avec zèle, les devoirs que nos fonctions nous imposent, dans Emile Bridrey, comme n. 4, 29-30.

${ }^{20}$ Cette assertion de Camille Poutbas, L'Instruction publique à Caen pendant la Révolution, in: Mémoires de l'Académie ... de Caen (1911) 124-125, s'est avérée invérifiable. 
peut se prévaloir du libre assentiment des citoyens. Sur un point particulier du nouveau régime, dans l'organisation des pouvoirs publics, les universitaires caennais manifestent leur préférence pour une réforme élargissant la prérogative royale ${ }^{21}$, pratiquement vidée de son sens par les Constituants.

Dans sa nature, le pouvoir de l'Assemblée est dénoncé comme «irrésistible», opprimant «les conceptions de l'esprit et les vœux de cœur* et, en dernière instance, divinisant l'Etat. L'Université soulève la contradiction entre le serment imposé par les Constituants et la Déclaration des droits de l'homme et du citoyen. En fait à une conception presque "rousseauiste, de la volonté générale, l'Université oppose la conception chrétienne selon laquelle ni la nation, ni le roi ne sont infaillibles: Dieu seul est infaillible, et son Eglise sur la terre. En mettant en cause, non seulement l'œuvre mais encore le pouvoir de l'Assemblée nationale, l'Université de Caen adoptet-elle la condamnation "intransigeante", voire *injuste ${ }^{22}$ du pape Pie VI dans son bref du 10 mars? En étendant ses critiques à l'ensemble du nouveau système, la déclaration entre-t-elle dans une pensée contre-révolutionnaire? D'un côté elle reconnaît que la Révolution met «la Nation à la place de Dieu* ${ }^{23}$. D'un autre côté, son souci de la prérogative royale ne l'empêche pas de rappeler que le roi n'est pas infaillible. Aussi peuton considérer qu'en dépit d'une mise en cause fondamentale, l'Université en tire une attitude conciliante en acceptant l'éventualité d'un serment restrictif ${ }^{24}$. Radicale en théorie, modérée en pratique, la Déclaration caennaise peut être susceptible d'interprétations diverses quant à son sens et à sa portée. En revanche le doute s'évanouit devant la condamnation formelle de la Constitution civile du clergé.

\section{B. Condamnation de la Constitution civile du Clergé:}

Dans sa condamnation de la Constitution civile du Clergé, l'Université revient sur son idée d'abus de pouvoir. L'Assemblée nationale s'est placée aau-dessus de ses pouvoirs ${ }^{25}$ par une série d'ignorances volontaires ou non.

$\left.1^{\circ}\right)$ Ignorance de l'antériorité des serments. Au premier évêque constitutionnel du Calvados, Claude Fauchet, qui, arrivant de Paris, devait déclarer que les scrupules

\footnotetext{
${ }^{21}$ L'Université de Caen, après l'édit de réforme de Louis XVI en 1786, détient depuis 1787 un portrait du roi dans sa bibliothèque. Ce tableau devait être brûlé en août 1792 devant les bâtiments universitaires: Camille Pouthas, comme n. 20, 30.

${ }^{22}$ Pour reprendre les adjectifs d'Albert Matbiez, Rome et le clergé français sous la Constituante (Paris 1911) 493.

${ }^{23}$ Albert Matbiez, comme n. 22, 446.

${ }^{24}$ Il faudrait ranger la Déclaration de l'Université de Caen dans l'sAnti-Révolutions, à la différence, par exemple, de l'arrêt du Parlement de Toulouse, qui, après la loi des 6-7 septembre 1790 supprimant les Cours souveraines, avait condamné l'ensemble de l'œuvre de l'Assemblée nationale: Philippe Wolff, Histoire du Languedoc (Toulouse 1967) 443. Sur la distinction entre Contre-Révolution et Anti-Révolution: François Lebrun et Roger Dupuy. Les résistances à la Révolution (Paris 1987) 469.

${ }^{25}$ On retrouve l'antagonisme entre conception rousseauiste et conception chrétienne. Pour Rousseau, l'Eglise ne peut s'opposer à la volonté générale, tandis que les Constituants unissent «au point de les confondre l'Eglise et l'Etat, Albert Matbiez, comme n. 22, 444.
} 
étaient «hors de saison. ${ }^{26}$, l'Université a, par avance, répondu qu'un parjure religieux est une apostasie.

$2^{\circ}$ ) Ignorance de l'antériorité de la liberté de l'Eglise sur l'autorité de l'Etat. Les lois ecclésiastiques ne peuvent se placer en aval des lois civiles, et l'Etat ne peut intervenir que comme instrument et non comme décideur (carte des évêchés et des paroisses, par exemple).

$3^{\circ}$ ) Ignorance de la nature de l'Eglise, dans laquelle les modifications disciplinaires ne sont pas indépendantes du dogme, "toujours immuable comme la vérité incréée».

$4^{\circ}$ ) Ignorance de la structure de l'Eglise, dans laquelle le pouvoir est exercé en premier par le pape, prince des apôtres, puis par les évêques. Y donner le pouvoir à des collèges électoraux civils, c'est s'abandonner à l'Etat.

Les universitaires caennais appuient leur condamnation par un système triplement référencé quant à l'objet des «réformes», la liberté de l'Eglise et le pouvoir dans l'Eglise. Quant à l'objet des réformes, l'Eglise en est seule juge. Sur ce point l'Université se réfère au bref que le pape Pie VI a envoyé le 10 mars précédent au cardinal de La Rochefoucauld, archevêque de Rouen, et aux autres prélats membres de l'Assemblée nationale, bien que ce bref n'ait pu être publié par l'évêque de Bayeux avant sa fuite pour l'étranger et bien que ce texte n'ait pas été publié par le gouvernement ${ }^{27}$.

Quant à la liberté de l'Eglise et à l'antériorité de ses lois sur les règlements de l'Etat, la déclaration caennaise s'appuie sur des auteurs gallicans, parmi les moins suspects au gallicanisme politique: Pierre de Marca et Le Vayer de Boutigny. En matière de droit le traité de Pierre de Marca, qui avait simplifié la polémique gallicane, était devenu une référence $^{28}$ où «se trouvaient codifiées et clairement exposées les maximes gallicanes. ${ }^{29}$. De son côté, le Traité de l'autorité des rois touchant l'administration de l'Eglise rédigé, à la demande de Louis XIV, par Roland Le Vayer de Boutigny, maître des requêtes, et resté sous forme de mémoire manuscrit jusqu'au XVIIlème siècle, apparut comme «l'œuvre la plus remarquable (...) véritable somme du système gallican ${ }^{30}$

Enfin, quant à la distribution du pouvoir dans l'Eglise, quant à la prééminence du

${ }^{26}$ Le 26 juin 1791 en l'église Saint-Etienne. Voir Eugène de Robillard de Beaurepère, Etudes sur l'histoire de la Révolution dans le Calvados (Caen 1922) 59.

${ }^{27}$ Rappelons que ce très long texte est une réponse de la Cour de Rome à l'Exposition des principes faite par les prélats membres de l'Assemblée nationale en octobre 1790.

${ }^{28}$ De concordia sacerdotii et imperii (Paris 1641). Sur Pierre de Marca et le De concordia, nous renvoyons à François Gaquère, Pierre de Marca (Paris 1932) 100-188.

${ }^{29}$ Henri-Jean Martin, Livre, pouvoirs et société à Paris au XVIIe siècle, tome 1 (Genève 1969) 185. Le traité De concordia est mis à l'Index en 1642. L'auteur se rétracte en 1647 reconnaissant qu'il a composé son livre «en magistrat plutôt qu'en évêque•, Franģois Gaquère, comme n. 28, 190.

30 René Taveneaux, Le catholicisme dans la France classique (Paris 1980) 509. Dans sa quatrième instruction à son fils, le chancelier d'Aguesseau recommande le traité de Le Vayer de Boutigny "pour se former une juste idée de la distinction des deux puissances». Edmond Préclin et Eugène Jarry, Les luttes politiques et doctrinales aux XVIIe et XVIIIe siècles (Paris 1955) 222. 
pape et aux attributs des évêques, l'Université se réfère deux fois à Bossuet ${ }^{31}$, une fois à la Déclaration des quatre articles de $1682^{32}$ et une fois à Fleury ${ }^{33}$.

Ainsi, à l'exception du Traité de Le Vayer de Boutigny, toutes les références sont tirées d'un gallicanisme ecclésiastique - essentiellement épiscopal et passablement modéré très éloigné, et des tendances presbytériennes et du gallicanisme parlementaire. Bossuet, en particulier, n'a-t-il pas insisté sur la distinction entre l'Eglise et l'Etat *avec une netteté que l'on ne retrouvera pas avant l'enseignement de Léon XIII. ${ }^{34}$, revendiquant hautement le droit de l'Eglise en face des magistrats du roi?

Au total, par sa déclaration, l'Université révèle deux alternatives: la patrie ou la liberté de conscience d'une part, la souveraineté nationale ou la liberté de l'Eglise d'autre part. Dans les deux cas, elle choisit le deuxième terme: liberté de conscience et liberté de l'Eglise. Telle est, dans ses grandes lignes, la Déclaration du 25 mai 1791. Quelle peut être la part des conditions locales? Pourquoi la crise des serments conduit-elle à Caen à cette manifestation de résistance? Telles sont les questions qu'il faut examiner avant de s'interroger sur les conséquences immédiates et lointaines de la déclaration. Rien, en effet, ne répond, dans ce texte, ni au stéréotype normand, ni aux traditions de l'Université de Caen. Le stéréotype normand se manifeste par la préférence pour le côté pratique des choses, au détriment du débat théorique, par le respect pour les autorités constituées plutôt que par leur contestation. Sous ce rapport la Déclaration de 1791 apparaît atypique. Rien n'y répond également aux traditions universitaires caennaises, fort peu originales. Protestante quand le protestantisme fut à la mode, janséniste à l'heure du jansénisme, l'Université aurait pu être *patriote" au temps des jacobins. La Faculté de théologie, elle-même, se montrait fort tolérante depuis l'enseignement cartésien du Père André au début du XVIIIème siècle; quant à la Faculté des droits et à la Faculté de médecine, elles étaient aux mains des laïcs qui donnent vingt signatures sur un total de quarante huit. Or, en 1791, la résistance de l'Université se manifeste d'une manière solennelle et étendue. Le refus du serment universitaire est presqu'unanime après rétractations: un agrégé aux droits, LebretonLamy, revient sur son serment et rejoint les cinq agrégés déjà insermentés avant le 7 juin ${ }^{35}$. Cette attitude révèle un corps professoral particulièrement scrupuleux et d'abord chez les professeurs titulaires, ceux qui risquent le plus. Au corps professoral s'ajoutent les signatures de quatre docteurs en théologie, puis de quatre autres ecclésiastiques, le tout dessinant un mouvement de résistance à la Révolution, encore plus net à Caen et dans le diocèse de Bayeux que dans le reste de la Normandie. Par sa déclaration, l'Université se hisse aux premiers rangs des résistances à la Révolution

\footnotetext{
${ }^{31}$ Exposition de la doctrine de l'Eglise catholique sur les matières de controverse (1668). Voir Aimé-Georges Martimort, Le gallicanisme de Bossuet (Paris 1953).

32 Declaratio cleri gallicani (1682). Voir Edmond Préclin et Eugène Jarry, Le lotte politiche et dottrinali nei secoli XVIIe XVIII, 2 vol. (Turin 1974-1975) 593-604 (le gallicanisme par Robert Darricau).

${ }^{33}$ Institution au droit canonique, recommandée par d'Aguesseau à son fils au même titre que le traité de Le Vayer: Edmond Préclin et Eugène Jarry, comme n. 30, 222.

${ }^{34}$ Aimé-Georges Martimort, comme n. 31, 702.

${ }^{35}$ Emile Bridrey, comme n. 4, 64-65.
} 
comme l'évêque de Bayeux venait de se montrer un des adversaires les plus intrépides de la Constitution civile du clergé.

Comment expliquer, localement, cet état de fait?

D'abord Mgr de Cheylus a imprimé un très fort mouvement local de résistance, ralliant un clergé qui ne l'estimait guère, y compris des curés jansénistes et universitaires! De leur côté, les Eudistes qui avaient tant contribué à contenir le jansénisme dans le clergé normand, ne se sont guère portés vers le système ultra-gallican et philosophique élaboré par l'Assemblée constituante. Sans doute le doyen de la Faculté des Arts, l'abbé de La Rue, est-il hostile aux Eudistes. Mais c'est l'un d'entre eux, François Lefranc, supérieur du séminaire de Coutances, homme de confiance de son évêque, qui place l'interprétation de la Révolution en marche sur un terrain encore peu exploré, rapprochant les nouvelles institutions de certains usages et symboles maçonniques ${ }^{36}$. En cultivant l'attachement au Saint-Siège les Eudistes se multiplient contre le serment $^{37}$. Aussi le clergé réfractaire, légèrement minoritaire aux diocèses de Rouen $(46 \%)$ et de Coutances $(46 \%)$, très minoritaire au diocèse d'Evreux $(36 \%)$, compte pour plus de $60 \%$ dans les diocèses de la moyenne Normandie: Bayeux $(61 \%)$, Lisieux $(63 \%)$, Séez $(62 \%)$. Or cette résistance cléricale importe pour l'Université de Caen. Sauf aux droits et en médecine, toutes les chaires de l'Université sont occupées par des ecclésiastiques; les principales dignités et fonctions sont assurées par des prêtres. Pierre Rouelle, curé d'Hermanville depuis 1786, petite paroisse de la plaine de Caen, est recteur. Professeur de grec au collège du Bois, ce prêtre, natif du Cotentin, avait été le rénovateur de la Société d'agriculture de Caen ${ }^{38}$. Natif de Caen, neveu du général Dugommier, régent du collège du Bois, Henry-François Coquille-Deslongchamps avait été recteur en 1779; depuis 1786 il est syndic-général. Egalement natif de Caen, le doyen de la Faculté des arts aux cours recherchés ${ }^{39}$.

La présence de ces prêtres caennais invite à s'interroger sur les conditions les plus locales. On trouve un clergé universitaire, de modeste extraction. Son gallicanisme est sur la défensive. En 1781 un bachelier en théologie a pu soutenir sa thèse sur l'Eglise sans l'hommage habituel à la Déclaration des quatre articles. L'omission fut certes remarquée par Jacques Lentaigne, curé de Saint-Sauveur à Caen, qui alerta le doyen de

${ }^{36}$ Fransois Lefranc explique la Révolution française par un double complot, jésuitique contre la monarchie et maçonnique contre l'Eglise. En rassemblant tout un ensemble d'informations dont certaines sont fantaisistes - il opère la confusion entre révolution et conspiration: Pierre Chevallier, Histoire de la franc-maçonnerie française, t. I (Paris 1974) 382-383; Marcelin Defourneaux, Complot maçonnique et complot jésuitique, in Annales historiques de la Révolution française (1965) 170-186. Sur François Lefranc: Charles Bertbelot du Chesnay, François Le Franc, in Catholicisme ... IV (1956) 1537-1538; Jules Blouet, Les séminaires de Coutances et d'Avranches (Paris 1936) 186-205; Jean Bindet, François Becherel (Coutances ${ }^{2} 1971$ ) 109-110.

37 Guillaume de Bertier de Sauvigny, Eudistes, in: Catholicisme ... IV (1956) 666-668; Charles Bertbelot du Chesnay, Eudistes, in: Dictionnaire d'histoire et de géographie ecclésiastiques XV (1963) 1331-1335. On n'oubliera pas cependant que l'abbé de La Rue, hypothétique auteur de la déclaration de 1791, était hostile aux Eudistes: Paul Milcent, Un artisan du renouveau chrétien au XVIIe siècle: Saint Jean Eudes (Paris 1985) 557.

${ }^{38}$ Un de ses élèves, Jacques Thierry, devait se cacher pendant la Révolution, avant de tenir un des principaux salons de la vie intellectuelle caennaise à l'époque de Napoléon.

${ }^{39}$ Frédéric Vaultier, Notice sur la vie ... de feu M. l'abbé de La Rue (Caen 1838). 
la Faculté de théologie. Si le candidat dut représenter sa thèse avec les références à la Declaratio cleri gallicani, l'incident n'en révéla pas moins un déclin du gallicanisme.

De son côté le jansénisme est bien présent à Caen où les controverses demeurent à l'époque de Louis XVI. Une partie de la ville semble janséniste comme plusieurs curés, tel Lentaigne déjà cité. Mais, sans abdiquer l'esprit critique, ces jansénistes caennais sont strictement royalistes ${ }^{40}$ dans une ville où Louis XVI a séjourné deux fois en 1786. Aussi un Lentaigne, très apprécié à Rouen comme à Caen, plusieurs fois recteur de l'Université, peut-il signer une déclaration à laquelle il a contribuée ${ }^{41}$.

Enfin on ne saurait oublier la présence à Caen d'une bourgeoisie protestante, à la tête de l'industrie textile du tissage: les "Messieurs de Caen *2. Ces notables protestants constituent sun exemple typique» d'une bourgeoisie d'affaires, ayant le goût de la sélection sociale, du secret et de l'autorité, avide de réformes lui donnant place dans l'Etat. En soutenant les idées nouvelles, elle peut se saisir des postes de direction que lui offrent les circonstances, avant de marquer sa faveur pour la Constitution civile du clergé. Le 17 juin 1790 plusieurs de ces notabilités protestantes ont fondé le Club des Jacobins pour défendre à Caen la Constitution et les décrets de l'Assemblée nationale. Après la condamnation par la papauté des aspects schismatiques, voire hérétiques, de la politique de la Constituante, il est fort plausible que l'Université de Caen ait voulu faire pièce au protestantisme local ${ }^{43}$. Ce qui est assuré, c'est, qu'au district de Caen, le recteur Rouelle dut laisser sa place à l'un de ces grands notables protestants, Mesnil qui devait marier sa fille à Lindet à l'époque de la Convention.

Quelles que soient ces conditions locales, sur le plan général «la conjoncture était propice à une explication de fond ${ }^{44}$. Les ratures mêmes du texte original de la Déclaration ${ }^{45}$ révèlent le soin mis à la rédaction de la protestation. Parlant de la *Nation*, le mot «anarchie» est biffé au profit de "philosophie désastreuse»; parlant du roi, le mot "hommage" est rayé au profit du mot «souvenirs». Le débat général porte bien sur les bornes du spirituel et du temporel, sur l'origine, la nature et l'exercice de la liberté de l'Eglise. La Constitution civile du clergé subordonne l'Eglise à l'Etat dans un système ultra-gallican et pseudo-richériste. Elle n'est pas un acte d'hostilité vis-à-vis de la religion chrétienne, mais elle est bien plus forte qu'une séparation de l'Eglise et de l'Etat. Alors que dans une séparation, seul l'Etat est laïcisé, la Constitution civile du clergé remet l'Eglise au pouvoir des laïcs, en fait aux mains des citoyens actifs et des électeurs $^{46}$. La Déclaration de l'Université de Caen représente donc pour des esprits habi-

40 Georges Vanel, Notes anecdotiques sur le jansénisme à Caen, in: Mémoires de l'Académie ... de Caen (1918-1920) 1-56.

${ }^{41}$ Emile Sevestre, La vie religieuse dans les principales villes normandes pendant la Révolution, 1787-1801, tome 1: Calvados (Caen 1945) 167.

42 Emile G. Léonard, La bourgeoisie protestante et sa position politique et religieuse du XVIIIe siècle à la Révolution, in: Assemblée générale de la Commission centrale et des comités départementaux 1939, I (Besançon 1942) 174.

${ }_{43}$ Emile Léonard, L'abbé de La Rue (Caen 1937) 6. Sur 37500 habitants, Caen compte au moins 2000 protestants.

44 Bernard Plongeron, comme n. 13, 197.

${ }_{45}$ Treize ratures approuvées sur le registre, Archives départementales du Calvados, D 83.

${ }^{46}$ Ce qui n'empêche pas l'abbé Plongeron d'interpréter la Civilis Cleri Gallicani constitutio par 
tués au gallicanisme et/ou au jansénisme un effort tout-à-fait considérable pour rappeler la nature et la structure du pouvoir de l'Eglise et dans l'Eglise. Bien que «respectueuse ${ }^{47}$ elle jette une lumière vive sur les abus de pouvoir commis au nom de la $\mathrm{Na}$ tion, de la Loi et du Roi. Ses auteurs ont une exacte conscience des dangers auxquels ils s'exposent, le refus du serment universitaire étant puni par la destitution. Après la fuite de leur chancelier, l'évêque de Bayeux, ils redoutent les malheurs qui peuvent frapper l'institution universitaire comme leur personne ${ }^{48}$. Mais ils n'hésitent pas à critiquer, voire condamner ce que la Révolution en marche a de "contraire au droit de la conscience ${ }^{49}$.

\section{Les conséquences de la Déclaration}

La Déclaration de l'Université de Caen déclenche des réactions rigoureusement contradictoires qui révèlent, à leur tour, la logique du conflit sur l'origine, la nature et l'étendue des pouvoirs respectifs de l'Eglise et de l'Etat ${ }^{50}$.

\section{A. Les conséquences immédiates:}

Bref laudatif du pape et fermeture de l'Université sont les conséquences immédiates de la Déclaration du 25 mai 1791.

En date du 9 juillet, le pape Pie VI répond au recteur et au syndic-général qui avaient expédié la déclaration en Cour de Rome, par un bref d'éloge et d'encouragement $^{51}$. Le pape insiste particulièrement sur l'opportunité et la solidité de la déclaration de Caen. Opportunité, après les brefs des 10 mars et 13 avril, comme après les

\section{Fortsetzung Fußnote von Seite 72}

le sacerdoce commun des baptisés et ... le deuxième concile œcuménique du Vatican: Bernard Plongeron, comme n. 13, 203. De son côté Timothy Tackett envisage l'élection des curés par les citoyens actifs et celle des évêques par les électeurs comme un procédé "démocratique*: La Révolution, l'Eglise, la France: le serment de 1791 (Paris 1986) 32.

47 Jacques Laffetay, Histoire du diocèse de Bayeux XVIIle et XIXe siècles, Tome II (Bayeux 1876) 228.

48 Expulsé de l'évêché de Bayeux le 3 avril 1791, il doit se réfugier d'abord à la campagne, puis s'enfuir pour l'étranger déguisé en marchand.

49 C. Pouthas, La constitution intérieure de l'Université de Caen au XVIIle siècle, in: Mémoires de l'Académie ... de Caen (1909) 34.

${ }^{50}$ Le conflit entre la Révolution française et l'Eglise catholique est en effet logique malgré son déclenchement circonstanciel: $R$. Laprat, Les libertés gallicanes sous la Révolution française et à l'époque moderne, in: Dictionnaire de droit canonique, VI (Paris 1957) 509-525: toute conciliation était impossible, 511. Ce qui différencie en effet la Révolution française des révolutions anglo-saxonnes antérieures, c'est précisément l'idée de régénérer l'homme par une société nouvelle; dès lors la révolution doit absorber le religieux dans le social et le politique: voir, entre autres études de François Furet, La Révolution dans l'imaginaire politique français, in: Le Débat 26 (1986) 173.

31 Le bref de Pie VI est connu à Caen vers le milieu de juillet 1791: Georges Lesage, Episodes de la Révolution à Caen (Caen, s.d.) 64. Voir son texte aux Archives départementales du Calvados, D 517 bis ou in Baicocana, II (1910) 48 et 83-85. Rappelons que les relations diplomatiques entre la France et le Saint-Siège étaient largement interrompues depuis mars 1791. 
censures portées par l'évêque de Bayeux sur la politique de l'Assemblée constituante. Quant à la solidité de la protestation universitaire, le pape l'analyse sur le plan moral et sur le plan intellectuel. Sur le plan moral, Pie VI approuve la position de l'Université dans la crise des serments: aucune révolution ne peut justifier un parjure. Sur le plan intellectuel, le souverain pontife approuve, avec non moins de vigueur, la distinction faite à Caen entre le droit de l'Eglise et le droit de l'Etat, sans relever les références à des ouvrages gallicans qui ont pu être à l'Index sauf rétractation comme chez un Pierre de Marca. Lorsque ce bref de Pie VI est connu à Caen vers la mi-juillet, l'Université, entre temps, avait été fermée.

A Caen, en effet, loin d'avoir un faible retentissement et d'être sans effet sur la marche des événements ${ }^{52}$, la Déclaration a provoqué des conséquences considérables. «La signature d'un grand nombre de professeurs laïcs ... et des agrégés des deux Facultés (droits, médecine) sembla donner un nouveau poids et plus de force aux sentiments exprimés dans les déclarations des ecclésiastiques. Tous les bons citoyens s'empressaient de se procurer la Déclaration. ${ }^{53} \mathrm{~A}$ la Faculté des droits, quatre professeurs sur cinq avaient signé la Déclaration; le cinquième, Alexandre, s'y rallia ensuite. Les signatures de prêtres affluent ${ }^{54}$. Remise au greffe du département du Calvados le 26 mai, la Déclaration y est lue le vendredi 3 juin. Tandis qu'à Paris elle est sévèrement jugée $^{55}$, à Caen, le directoire du département, le 10 juin, arrête la condamnation du texte, la destitution et l'interdiction des signataires, la fermeture des registres et des locaux $^{56}$. Le 18 juin l'Université de Caen est fermée par les soins de la Municipalité. Le lendemain la manifestation prévue par le club des Jacobins est interdite ${ }^{57}$. La rudesse de la réaction révèle combien les administrations locales sont ulcérées par la résistance de l'Eglise: multiples protestations de l'évêque de Bayeux, protestations du clergé de Caen et du diocèse de Bayeux, démission du maire de Caen, démission du premier évêque constitutionnel, abstentions grandissantes ${ }^{58}$ aux élections épiscopales et curiales et, brochant sur le tout, la déclaration de l'Université! Car c'est bien à la déclaration, plus qu'au refus de serment que s'attache l'arrêté du département, y dénonçant

52 Camille Pouthas, comme n. 20, 121-233.

53 Observation de Laurent Esnault, avocat au ci-devant bailliage de Caen dans: Georges Lesage, comme n. 51, 59.

54 Archives départementales du Calvados, D 83, fol. 46 par exemple. Adhèrent à la Déclaration les clergés des paroisses Saint-Sauveur et Saint-Jean de Caen, etc.

55 Au Comité d'instruction publique de l'Assemblée nationale, la Déclaration de Caen est considérée comme une adiatribe factieuse: Louis Liard, L'Enseignement supérieur en France 1789-1889, tome 1 (Paris 1888) 122.

56 Les 36 administrateurs du département ont été élus en juin 1790 par 499 électeurs sur 594 : Robert Patry, comme n. 16, 197.

57 Il avait été prévu de promener un âne coiffé du bonnet du recteur. A la queue de l'animal aurait été attachée la déclaration. Sur ses flancs auraient été placées les listes des signataires.

58 Pour élire l'évêque du Calvados, le nombre des votants diminue de 400 à 250 entre le début mars et la mi-avril 1791: Fauchet est élu par 152 voix. L'élection des curés de Caen dure du 10 avril au 20 mai avec 75 électeurs au début, 50 à la fin: Emile Sevestre, comme n. 15, 172-178 et Robert Patry, comme n. 16, 245. 
une «coalition séditieuse ${ }^{39}$. Ainsi se trouve anéantie l'Université de Caen alors que les autres centres fonctionnent jusqu'en 1793. Quand, le 4 août 1791, le Ministre de l'Intérieur signale aux autorités départementales qu'elles avaient à destituer et à remplacer les universitaires insermentés et non à fermer l'Université, il est trop tard: le Calvados anticipe - de plus de deux ans et demi -, la marche des événements.

\section{B. Les conséquences ultérieures:}

En 1800 le bref de Pie VI à l'Université de Caen - et sa traduction française - sont imprimés en Angleterre par les soins de l'abbé Vasse, ci-devant professeur de théologie de la Faculté de Caen ${ }^{60}$. Toutefois les publications locales de l'époque napoléonienne semblent ignorer 1791 malgré la restauration de Facultés à Caen ${ }^{61}$.

Il faut attendre la Restauration pour voir la Déclaration universitaire et le Bref faire l'objet d'une publication officielle de l'Académie de Caen, à la tête de laquelle les milieux ultra-royalistes ont fait porter, en 1822, l'abbé Jamet ${ }^{62}$. Dans sa séance du 24 novembre 1824, le Conseil de l'Académie de Caen décide de réimprimer ensemble la Déclaration de l'Université et le Bref du pape ${ }^{63}$. En présentant ces documents au nouveau roi, Charles $\mathrm{X}$, le recteur Jamet obtient du monarque un souvenir. Le comte d'Artois avait lu la déclaration de 1791 et avait écrit au recteur de l'Université de Caen pour le féliciter «d'un acte de loyauté si noble et si courageux * ${ }^{64}$. Mais le recteur Jamet ne put obtenir ni la restauration de la Faculté de théologie, ni la restauration de la Faculté de médecine. Désormais la déclaration et le bref vont passer des archives actives aux archives historiques.

Sous le Second Empire un historique de l'ancienne Faculté des droits évoque la crise de $1791^{65}$. Son auteur voit adans cette protestation courageuse, demeurée, selon nous, pour nos anciennes écoles caennaises, un titre à l'estime de la postérité ${ }^{66}$. L'abbé Laffetay, historiographe du diocèse de Bayeux aux XVIIle et XIXe siècles, y voit une déclaration "respectueuse ${ }^{67}$.

Au début du XXème siécle $C$. Pouthas n'est pas loin de considérer la déclaration de 1791 comme une simple diatribe contre-révolutionnaire. Sans doute reconnaît-il qu'elle est prise pour défendre le droit de la conscience, mais il n'en donne aucune

59 Point de vue partagé au XXème siècle par Emile Bridrey qui y voit une *insurrection contre la lois: Emile Bridrey, comme n. 4, 66.

${ }^{60}$ Essai ... sur la conduite à tenir par le clergé fidèle lors de sa rentrée en France (Londres 1800).

${ }^{61}$ La Notice sur M. Chibourg, publiée par P. B. Thierry (Caen 1807) ne dit rien sur la Déclaration de 1791 alors que Chibourg, ancien recteur et professeur à la Faculté de médecine est un des signataires et que la famille Thierry est liée au dernier recteur, l'abbé Rouelle.

62 Solide biographie de Georges-Abel Simon, La vie de l'abbé Pierre-François Jamet (Caen ${ }^{2} 1959$ ).

${ }^{63}$ Académie royale de Caen: Déclaration de l'Université de Caen au sujet des lois des 22 mars et 17 avril 1791, concernant le serment prescrit aux fonctionnaires publics; et bref du pape Pie VI, adressé aux recteur et syndic-général (Caen 1824).

${ }^{64}$ Georges-Abel Simon, comme n. 62, 178.

${ }^{65}$ Jules Cauvet, Le collège des droits de l'ancienne université de Caen (Caen 1858), extrait des Mémoires de la Société des Antiquaires de Normandie, XIII, 447-575.

${ }_{66}$ Jules Cauvet, comme n. 65, 159-160.

${ }_{67}$ Voir note 45 supra. 
analyse, en minimise la portée considérant qu'elle n'eut qu'un faible retentissement et qu'elle n'eut aucun effet sur la marche des événements ${ }^{68}$.

Etudiant l'ancienne Faculté des droits, Emile Bridrey admet que la fermeture de l'Université fut aune mesure d'ordre collectif extrêmement grave. ${ }^{69}$. Mais la Déclaration, "véritable insurrection contre la loi ${ }^{70}$ lui apparaît *un peu trop comme un sermon, ... pas assez comme un plaidoyer ${ }^{71}$. En sens inverse l'analyse redevient favorable chez Henri Prentout qui conclut: ¿Quoique l’on puisse penser des événements, cet acte, le dernier acte collectif de l'Université, par l'élévation des pensées, la noblesse des sentiments, lui fait honneur. ${ }^{72}$ Après quoi la Déclaration de 1791 disparaît de l'historiographie $^{73}$ à deux exceptions près ${ }^{74}$.

Au terme de cette communication, au risque de tomber dans le travers habituel du genre monographique qui attribue volontiers à son objet une cohérence et une importance plus étendues qu'en réalité, marquons la valeur documentaire de la Déclaration de l'Université de Caen dans le mouvement des idées et dans l'histoire de l'institution universitaire à la fin du XVIIle et au début du XIXe siècle. Son étude apporte deuX confirmations, deux infirmations et une invitation.

Deux confirmations? D'abord, malgré sa modération pratique, la Déclaration de 1791 confirme le caractère logique du conflit entre la Révolution constituante et l'Eglise catholique. Au début du XXe siècle Albert Mathiez rappelait que la Révolution française est une religion, d'où son incompatibilité première avec l'Eglise. De nos jours François Furet insiste sur la différence entre Révolution française et révolutions anglo-saxonnes antérieures. La Révolution française recherche une totale régénération de l'homme dans une société nouvelle, régénération qui absorbe le religieux dans le

68 Voir note 49 supra.

69 Emile Bridrey, cómme n. 4, 73.

70 Emile Bridrey, comme n. 4, 66.

71 Nous citons plus largement Emile Bridrey, comme n. 4, 59. „Cette Déclaration est, incontestablement, au point de vue moral, un acte courageux et digne; c'est aussi, dans la forme, un morceau d'une belle tenue littéraire, encore qu'elle apparaisse un peu longue peut-être et par endroits quelque peu verbeuse. Seulement, quand on la considère au point de vue du fond, l'argumentation n'en est rien moins qu'habile. Les rédacteurs ont appuyé beaucoup trop sur les considérations d'ordre théologique et cependant assez fortes, qu'ils auraient pu tirer, avec un peu plus d'adresse, de l'imprécision du texte des décrets et de l'embarras avoué du législateur lui-même, pour justifier leur refus. Si l'on veut d'un mot notre opinion, la Déclaration est un peu trop un sermon, elle n'est pas assez un plaidoyer. Et pour cela même, autant nous sommes portés à y reconnaître la main des professeurs de la Faculté de théologie, autant il nous paraît peu vraisemblable que leurs collègues de la Faculté des droits aient collaboré, même en second plan, à une argumentation en définitive maladroite pour la thèse soutenue..

72 Henri Prentout, comme n. 4, 185.

${ }^{73}$ Si le manque de place pourrait expliquer son absence de l'Histoire de la Normandie d'Emile Léonard (Paris 1944), cette raison est sans valeur pour les pages, pourtant remarquables, de Henry Contamine, La Normandie du XVIIe au XXe siècle, in: Les Etudes normandes: exposés et méthodes (Bayeux et Caen 1944) 247-252. Jean Vidalenc n'en souffle mot dans sa synthèse sur la période révolutionnaire où, il est vrai, la dimension religieuse de la crise est systématiquement sous-estimée: Michel de Boüard, Histoire de la Normandie (Toulouse 1970) 392-404.

74 Dans une remarquable évocation de la crise des serments, allusion à la déclaration par Paul Longuet, Caen dans la tourmente révolutionnaire, in: Gabriel Désert, Histoire de Caen (Toulouse 1981) 181. Voir également Robert Patry, comme n. 16, 259-260. 
social et le politique. Dans la réorganisation que la Nation souveraine opère dans l'Eglise, cette dernière peut-elle se laisser absorber sans se dénaturer, avec ses pasteurs devenant des administrateurs élus par les citoyens actifs et les électeurs? En face de ce système hybride - encore plus philosophique que presbytérien - les gallicans doivent réévaluer et les frontières entre l'Eglise et l'Etat et le pouvoir du Saint Siège, car, répétons-le, la pierre d'achoppement est bien l'origine, la nature et l'étendue du pouvoir. Le débat, en 1791, n'a rien de théorique, les universitaires y jouent leur place et l'Université son existence. Cette résistance de l'institution au pouvoir irrésistible de l'Assemblée constituante peut être interprétée comme un élément révélateur de sa vitalité $^{75}$. Par ailleurs la déclaration de Caen confirme le rôle du gallicanisme et du jansénisme dans la résistance à la Révolution, et non pas seulement comme origines de la Constitution civile du clergé ${ }^{76}$.

Deux infirmations. La Déclaration de 1791 infirme l'idée selon laquelle la Révolution aurait été relativement tranquille dans une province comme la Normandie avant la chouannerie. En fait la protestation universitaire s'inscrit dans un vaste mouvement de résistance des milieux ecclésiastiques normands contre la Constitution civile du clergé: la déclaration des curés de Caen annonce la déclaration de l'Université. En face la réaction du département du Calvados ou celle des jacobins locaux révèlent l'ardeur du parti patriote. Révolution, Contre-Révolution, Anti-Révolution? La Déclaration de Caen n'entre pas dans une Contre-Révolution aristocratique, ni dans une Anti-Révolution populaire. La multiplication des concepts et, en particulier, la distinction de nature sociale, proposée par C. Lucas en 1984, reprise par Claude Mazauric en 1985, ne semble pas ici opératoire ${ }^{77}$. Guère utile non plus l'idée de dérapage. Quant au pouvoir, le dérapage c'est précisément la Révolution de 1789. La Déclaration de Caen marque la fin d'un processus de rupture politico-religieux. Elle apporte une deuxième infirmation: non, l'Ancien Régime ecclésiastique et universitaire ne s'efface pas de lui-même, miné par les abus et les privilèges. La Déclaration de l'Université de Caen révèle au contraire la capacité de résistance d'une institution mixte, à la charnière de l'Etat et de l'Eglise. L'idée de sclérose ne peut être retenue pour une institution capable d'une pareille résistance ${ }^{78}$.

Une double invitation enfin. A s'interroger d'abord sur l'attitude comparée des universités en face de la Révolution car le livre de Liard est, sur ce point, insuffisant et la synthèse la plus récente sur l'histoire des universités en France est muette sur la crise

75 «L'Université de Caen, à la fin du XVIIle siècle, était florissante,, Henri Prentout, comme n. 4, 189.

${ }^{76}$ Edmond Préclin, Les jansénistes du XVIIIe siècle et la Constitution civile du clergé (Paris 1928); Edmond Préclin et Eugène Jarry, comme n. 32.

77 Il faudrait distinguer une Contre-Révolution nobiliaire et cléricale et une Anti-Révolution populaire: François Lebrun et Roger Dupuy, comme n. 24, 469. Mais la Déclaration de l'Université de Caen n'est pas œuvre populaire, sans être pour autant favorable à une restauration intégrale de 'Ancien Régime.

${ }^{78}$ L'Université de Caen, outre l'expédition à Rome, avait expédié sa Déclaration à d'autres universités, notamment Strasbourg: Louis Liard, comme n. 7, 122. En comparaison, voir la brève déclaration de la Faculté de théologie d'Aix-en-Provence le 28 mai 1791: Louis Liard, comme n. 7 , 384-390. 
révolutionnaire. A s'interroger ensuite sur les conséquences d'un anéantissement, précoce à Caen, ultérieur ailleurs, des universités. En privant la France de ces centres de culture religieuse et intellectuelle, la Révolution ne l'a-t-elle pas mise en état d'infériorité au XIXe siècle au regard du reste de l'Europe, notamment de l'Europe centrale ${ }^{79}$ ?

\section{Zusammenfassung des Referates von Jean Laspougeas}

Die Geschichte der Universitäten spiegelt sich in der Geschichte der Revolution und der Gegenrevolution wider. Wir werden dies an Kritik und Verurteilungen, die die Universität von Caen gegen das Werk der Constituante vorgebracht hat, zu zeigen versuchen.

Die Universität von Caen wurde als erste geschlossen. Dies geschah nicht wie bei den anderen im Sommer 1793, sondern bereits im Frühjahr 1791. Ursache der hastigen Schließung und vorzeitigen Auflösung war ein Protest der Universität gegen das Werk der „Assemblée nationale“, der in Form einer Denkschrift ausführlich begründet wurde und sofort in Druck erschien (25. Mai 1791).

Die Universität Caen brachte unter dem Eindruck eines einschränkenden Eids auf die Zivilkonstitution eine vernichtende Kritik des politischen und sozialen Werks der Constituante vor. Diese Kritik stützte sich einerseits auf den unvollendeten und unerprobten Charakter der neuen Einrichtungen, als sich eine ,anarchie sauvage et antisociale“ zu entwickeln begann, und auf der anderen Seite auf den unterdrückenden Charakter der „Assemblée nationale“, deren „pouvoir irrésistible“ die eigene Deklaration der Menschen- und Bürgerrechte ins Gegenteil verkehrte. Schließlich schloß diese Kritik auch die vermeintliche Unfehlbarkeit der Macht des Staates ein: Weder die Nation noch der König seien unfehlbar, so die Ansicht der Universität. Die einzige Unfehlbarkeit sei diejenige Gottes und seiner Kirche, die ihm in seinem Geist diene.

Die Universität fügte diesen grundsätzlichen Kritikpunkten eine strikte Verurteilung der neuen Kirchenordnung hinzu. Dabei vertrat sie eine gänzliche Ablehnung jeglichen Eids auf die Zivilverfassung des Klerus, was sie sowohl mit dem Vorrang und der Vorherrschaft der Religion über jegliche Revolution und des Geistlichen über das Weltliche begründete, als auch mit den Machtverhältnissen in der Kirche. Aus dem Vorrang der Religion über die Revolution ergebe sich, daß die Forderung des Staates einen Mißbrauch der Macht und eine Gefahr des Abfalls vom Glauben darstelle. Ebenso könnten die Veränderungen in der Lehre der Kirche nicht getrennt werden vom „dogme toujours immuable comme la vérité incrée“. Der Vorrang des Geistlichen über das Weltliche beweise, daß - wenn der Katholizismus in Frankreich die Religion des Staates sein solle - der Staat keine Autorität habe, die Kirche zu reformieren, es sei denn, er stelle sich ,au-dessus de ses pouvoirs“. Das bürgerliche Gesetz könne dem kanonischen Recht folgen, ihm jedoch nie vorausgehen. Schließlich zeige die Verteilung der Kräfte der Kirche, daß der Papst die Macht ohne Ausnahme in Händen habe und diese Macht sich nur beim Bischof wiederfinde, für den die Priester nur Kooperatoren sein könnten.

79 Henri Prentout, comme n. 4, 190. 
Die Universität von Caen erklärte in bezug auf die unannehmbare Zivilkonstitution des Klerus - nachdem sie das Gesetz verurteilt hatte -, daß sie sich an die Entscheidung und das Verhalten des Papstes und der französischen Bischöfe halten wolle. Da das Gesetz vorsah, Professoren, die den Eid verweigerten, zu ersetzen, rief die Deklaration der Universität eine brutale Reaktion der Behörden hervor. Zweifellos konnten diese in der Protestnote nur eine beißende Kritik an der Zivilkonstitution des Klerus sehen. Da die Behörden ohnehin schon erbittert waren über den massiven Widerstand des Klerus - und besonders des städtischen Klerus - und wegen des Kampfes des Bischofs von Bayeux gegen die Zivilkonstitution, erbittert auch wegen der Schwierigkeiten bei den Bischofs- und Pfarrerwahlen, so sahen sie hierin mehr als nur eine einfache Eidverweigerung, nämlich eine Verschwörung, einen echten Aufstand gegen die nationale Souveränität. Sie befahlen die Schließung der Universität. Während die anderen Universitäten bis zum Sommer 1793 bestehen blieben - oder überlebten -, vernichtete die Revolution die Universität von Caen im Juni 1791.

Zur selben Zeit, am 9.Juli, richtete Papst Pius VI. an die Universitäten ein Breve, in dem er insbesondere für die notwendige Unterscheidung zwischen der Macht des Staates und den Rechten der Kirche dankte.

Auf ihre Weise beansprucht diese Deklaration sowohl durch die Schwere der Kritik, die gegenüber der Constituante vorgetragen wurde, als auch durch die Reaktion der Revolutionsbehörden und des Heiligen Stuhls ihren Platz in der Geschichte der Revolution und der Gegenrevolution.

\section{Diskussion zum Referat von Jean Laspougeas}

\section{Leiter: Guillaume de Bertier de Sauvigny}

Einleitend hob $G$. de Bertier de Sauvigny anerkennend die Bereitschaft von J. Laspougeas hervor, bei der Beschäftigung mit der Französischen Revolution den Pariser Rahmen zu verlassen. Auf die Frage von $P$. Stadler nach dem Verhalten der übrigen Universitäten führte der Referent aus, daß damals in Frankreich 23 Hochschulen existiert hätten, von denen aber nicht alle die fünf traditionellen Fakultäten besessen hätten. Die Universität von Caen habe versucht, ihren Protest auch auf andere Universitäten auszuweiten, worin die Constituante Aufruhr gesehen habe. Auch die Theologische Fakultät von Paris habe im September 1791 eine Protestnote herausgegeben, was ihre Schließung zur Folge gehabt habe. Diese Initiative wäre aber nur von dieser Fakultät getragen gewesen, während in Caen die gesamte Universität protestiert habe. Über die Haltung der übrigen Hochschulen ist nach Auskunft von J. Laspougeas noch nichts bekannt.

M. Botzenhart interessierten besonders die materiellen Auswirkungen der Entscheidungen der Nationalversammlung auf die Universitäten. Der Referent stellte in seiner Antwort die These auf, das Gesetz, das den Kirchenbesitz zur Disposition der Nation gestellt habe, habe die "Schutzklauseln“ zugunsten der dem Unterricht gewidmeten Einrichtungen bereits vorweggenommen. So seien weder die Universität von Caen 
noch andere Universitäten, die die Zivilkonstitution des Klerus abgelehnt hätten, ihrer Einnahmen beraubt worden.

Auf den im Vortrag erwähnten François Lefranc ging G. de Bertier de Sauvigny ein und bezeichnete ihn als einen Vorläufer von Abbé A. de Barruel (1741-1820). Dieser habe die Revolution auf ein freimaurerisches Komplott zurückgeführt (Mémoires pour servir à l'bistoire du Jacobinisme, erschienen in Hamburg zwischen 1797 und 1799). Ferner wären zwei andere Protagonisten von 1791, Gervais de la Rue (1751-1835) und Mars, 1816 vom ultra-royalistischen Präfekten der Restauration als gefährliche Personen betrachtet worden. Hierzu ergänzte J. Laspougeas, der Rektor Mars sei 1823, während eines Konflikts zwischen drei Unterzeichnern des Protestes von 1791, seiner Funktion enthoben worden. Benutzt worden sei dies als Argument auch noch 20 Jahre später.

Für J. Tulard entstand der Konflikt, der durch die Zivilkonstitution des Klerus hervorgerufen wurde, dadurch, daß die Constituante das Gesetz schuf, ohne den Papst vorher konsultiert zu haben. Daran schloß sich die Frage an, wie die Universität von Caen auf die Verstaatlichung der Kirchengüter reagiert habe. Der Referent zeigte, daß die Universität von Caen vor allem die Ausdehnung der Staatsmacht auf die Kirchenorganisation verurteilen wollte, auf die Konfiskationen selbst aber nicht eingegangen sei. Die Universität habe die Constituante beschuldigt, sich Rechte angeeignet zu haben, die die Wähler ihr übertragen hätten. So wäre es weder im Machtbereich der Generalstände gelegen, eine Konstitution für den Staat zu schaffen, noch die Beziehungen zwischen Kirche und Staat zu reglementieren. In diesem Streit habe die Souveränität der Nation der Freiheit der Kirche gegenübergestanden. 